\title{
A Hybrid Method of Face Feature Extraction, Classification Based on MLBP and Layered- Recurrent Network
}

\author{
Aws Al-Qaisi ${ }^{*}$, Mokhled S. AlTarawneh ${ }^{2}$, AbdElRahman ElSaid ${ }^{3}$ Ziad Alqadi $^{4}$ \\ ${ }^{1}$ Department of Communication Engineering, Faculty of Engineering Technology, Al-Balqa Applied University, Amman, \\ 11134, Jordan \\ ${ }^{2}$ Computer Engineering Department, Faculty of Engineering, Mutah University, Mutah, 61710, Jordan \\ ${ }^{3}$ Golisano College of Computing and Information Sciences, Rochester Institute of Technology, Rochester 14623, USA \\ ${ }^{4}$ Computer Engineering Department, Faculty of Engineering Technology, Al-Balqa Applied University, Amman 11134, Jordan
}

Corresponding Author Email: aws.alqaisi@ bau.edu.jo

https://doi.org/10.18280/ts.370402

Received: 11 June 2020

Accepted: 12 August 2020

\section{Keywords:}

feature extraction, $M L B P$, classification, $L$ RNN, Quasi-Newton Back propagation

\begin{abstract}
Face feature extraction and classification is an attracting research area for its various applications. This paper proposes a hybrid technique based on modified local binary pattern (MLBP) and Layered-Recurrent neural network (L-RNN) to recognize the human faces. The proposed MLBP algorithm reduces the dimensions of extracted face images features. The classification process is conducted using L-RNN. The quasi-Newton back propagation algorithm is used to train the L-RNN. The proposed hybrid technique is examined on MUCT database and the performance analysis of different ANN techniques shows that the Hybrid technique is robust and has superior performance over conventional methods. It achieves the best classification rate of $98 \%$.
\end{abstract}

\section{INTRODUCTION}

Face recognition technology has the ability to identify individuals from a bunch of pictures or a video stream. This Technology is utilized in varied range of applications, such as human identification, access control and security investigation. The core of any biometric system depends on the accurate extracting features and classification, thus facial features detection and classification are a major concern in various applications such as face recognition, human computer interaction, surveillance systems, facial animation and facial expression [1]. About 300 papers focused on general facial feature extraction regarding different methods, some works applied handcrafted features, other employed statistical learning works reviewed by Wang et al. [2]. A contour-let transform with low and high frequency coefficient was used in several scales and angels, they used frequency coefficients as feature vector which processed by principle component analysis (PCA) for vector reduction to be adopted as the face classifier [3]. Scale and invariant rotation were used as a local feature detector. Thus feature extraction is done using speed up robust features (SURF) method and tags are arranged for the image utilizing adjacent neighbors [4]. SURF technique depends on interest point detection; its features descriptor is depending on the summation of the Haar wavelet response nearby the desired point. The limitations of this technique are gray scale images consideration and poorness approximation of key point orientation. Invariant features based on scale, invariant rotation and illumination was extracted using a scale invariant feature transform (SIFT) [5]. SIFT technique suffers from high dimensionality at matching and lagging in computational speed. A deep convolutional neural network (DCNN) technique as proposed as a feature extraction platform for feature fusion [6]. DCNN used two nets (Clarifai and $\mathrm{VGG}$ ) to get a convolution features, these features were fused to obtain more compact feature representation. A face recognition system depending on Gabor filter for global and local facial feature extraction was proposed [7]. Their extracted features were combined when the local are represented and the global are not clear. Thus, features are fused to construct a vector of descriptor features. The limitation of Gabor feature extractor is the enormous dimensionality of the features vector. A combination of principle component analysis and Eigen-face for extracting human face was proposed by Erwin et al. [8]. This method is limited to scale. Hence, preprocessing step is needed for scale normalization. A local binary patterns (LBP) method for image analysis was reviewed by Huang et al. [9]. It is used to represent local structure of image for the sake of image analysis. This method is resistive to the impact of lighting and useful for texture analysis [10]. LBP is suffering from heavy computational load. Several techniques of feature selection and extraction for facial recognition systems were studied and investigated by Lajevardi and Hussain such as local binary pattern (LBP), log Gabor filter and higher-order local autocorrelation (HLAC) [11]. Al-Qaisi et al. [12-14] analyzed several techniques which are utilized for texture features extraction of color images. These features can be utilized as a principal key to recognize images. In this paper, we proposed a modified local binary pattern approach to extract features of facial color image. The vector dimension of extracted features is reduced and can be used as an input to a neural network for training, classification and testing. Artificial neural networks ANN algorithms are machine learning techniques that are designed as an approximation of human brain model. ANNs have been widely used to overcome signal processing 
challenges such as speaker identification, face recognition, and classification difficulties $[15,16]$. Researchers have proposed many types of artificial neural networks to recognize a human face [17]. Principle component analysis might be accurate because it is used prior to the utilization of ANN to decrease the dimensionality of the data to get rid of the feature with less variance which would help in the training process and overcome the high variance problem, while the recognition rate equal 90\% [18]. Convolution neural networks algorithm is also used for face recognition. It might simple to use but not that easy to implement, especially to tune its hyper parameters during training. It helps to reduce classification errors between neighborhood classes and gives high performance. However, if the number of classes increases the recognition rate become quite low [19]. In addition, a radial basis function neural network is recommended to train and classify faces. It gives high recognition accuracy rate [20]. The face recognition accuracy rate using Bilinear Convolutional Neural Networks (Bilinear CNNs) is around $80 \%$ to $90 \%$ [21]. Using back propagation neural network for face recognition gave more than $85 \%$ accuracy rate. However, it has been found that the radial basis neural network was better than back propagation algorithm in terms of accuracy for face recognition [22, 23]. Retinal Connected Neural Network (RCNN) can recognize from $79 \%$ to $90 \%$ of faces in the data sets consists of 130 faces [24]. Rotations Invariant Neural Network (RINN) algorithm recognizes faces which have different degrees of rotation with accuracy rate varying from $85 \%$ to $95 \%$ [25]. Evolutionary optimization of neural networks is proposed for face recognition and detection. This technique merges evolutionary calculation and gradient-based learning and acts more rapidly than conventional algorithms without loss of accuracy [26]. Multi-Layer Perceptron (MLP) neural network is proposed for facial recognition applications. Compared to CNNs, MLP technique increases the effectiveness and precision of recognition process, due to assembling the neural network in a manner that is efficient in ignoring the majority of the nonfacial patterns [27]. The hybrid method of Wavelet transform and $\mathrm{ANN}$ is proposed to recognize a human face. It gives the lowest training period, excellent performance, and high recognition levels. However, the most discriminatory features cannot be extracted using the direct utilization of wavelet coefficients due to the meaningless data which can be found in the coefficients of wavelet [28].

The primary contribution of this paper lies in proposing a novel technique that merges the advantages of MLBP and LRNN.

This paper is organized as follows. In section 2 , the proposed hybrid method will be presented. Section 3will analyze and discuss the results of proposed technique. Section 4 will conclude the paper.

\section{PROPOSED HYBRID METHOD}

The proposed hybrid method relies on merging MLBP and LRNN as shown in Figure 1. The proposed algorithm is composed of three key sections: firstly; a pre-processing phase which consists of face image resizing and reshaping from three dimensional matrixes into one raw vector. Secondly; applying MLBP operator on reshaped face image which extracted the features of each face image and adding these features in one raw matrix. This features data base matrix contains all features of face images in the sense that each image represented by four features. Finally, LRNN is constructed to train and classify the extracted features.

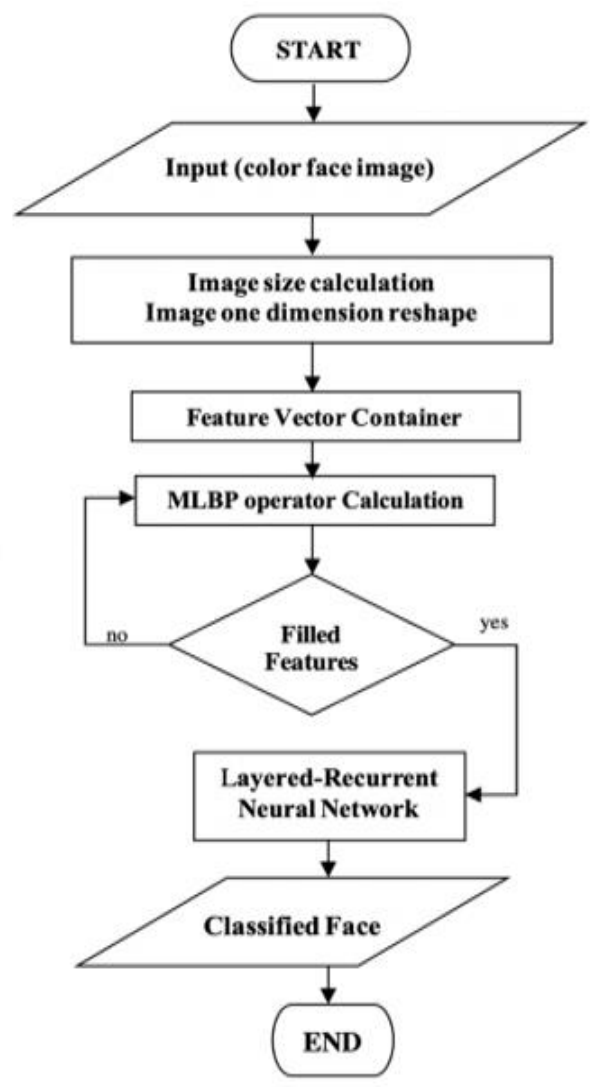

Figure 1. Proposed hybrid method

\subsection{MLPB feature extraction}

The extraction of features is a mechanism of dimension reduction which reduces the initial set of data toward more convenient parts for processing. In this manner treating color images was on the way of reshaping color image matrix into vector shape or one dimensional array, i.e. image with size (x1, $\mathrm{x} 2, \mathrm{x} 3$ ) a $3 \mathrm{D}$ image to be resized into $\mathrm{x} 1$ by $\mathrm{x} 2$ by $\mathrm{x} 3$ production then reshaping a $3 \mathrm{~d}$ matrix into $1 \mathrm{~d}$, this initialization step to use benefits of computation time reduction. Thus, previous steps were a construction preparation for feature extraction using modified local binary pattern approach (MLBP) which is pre-locating version of local binary pattern method [29], local binary pattern (LBP) [7, 30-32] is a strong local descriptor since it can efficiently represent the structural information of an input image to produce unique local features. A proposed approach used pixel intensity location comparison on 4 neighbors of pixel intensity values $(i-1, i+1, i-2, i+2)$ as shown in Figure 2.

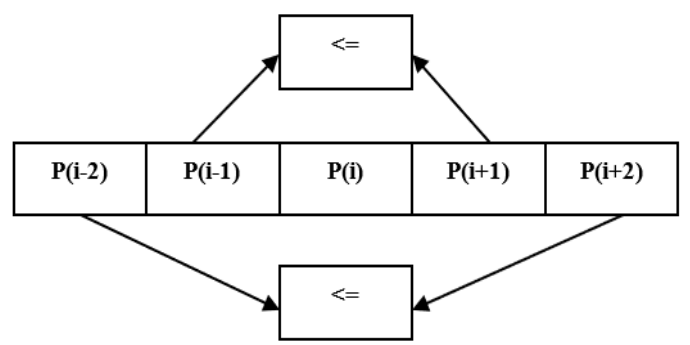

Figure 2. MLBP operator calculation (for each pixel) 
The MLBP feature extraction method working based following pseudo code

Algorithm 1. MLBP pseudo code

\author{
\#MLBP pseudo code \\ BEGIN \\ INPUT (face_image) \\ array $\leftarrow$ reshape into $1 \mathrm{D}$ array (face image) \\ Features matrix initialization by zeros \\ Counter i \\ WHILE counter $<=4$ \\ Features[i] $=$ Pixel_Intensity_Check(array) \\ Counter++ \\ ENDWHILE \\ END
}

\subsection{Classification using Layered recurrent neural network}

Classification is an essential stage in face recognition system. When the features are identified, they are used as an input to the classifier and after that the features will be classified using a neural network. L-RNN has been effectively implemented in numerous complex areas for example image processing, optimization, and industrial application [33, 34]. The initial edition of L-RNN was proposed by Elman [35]. Comparing with static or feedforward networks, the gradients in recurrent neural networks are dynamic as the feedback loop in the hidden layer is taken into account during the training and learning process on the basis of layer recurrence structure. Hence, every outcome in the hidden layer relies on both the measurement of the present and previous time step. As a result, the latest output will rely on both past and present input data. Consequently, during the training process, the amount of error among existing and expected outcome is reduced by gradient descent, where the weight values are progressively adjusted in a way that minimize this error as quickly and efficiently as possible. Figure 3 shows the construction of L-RNN.

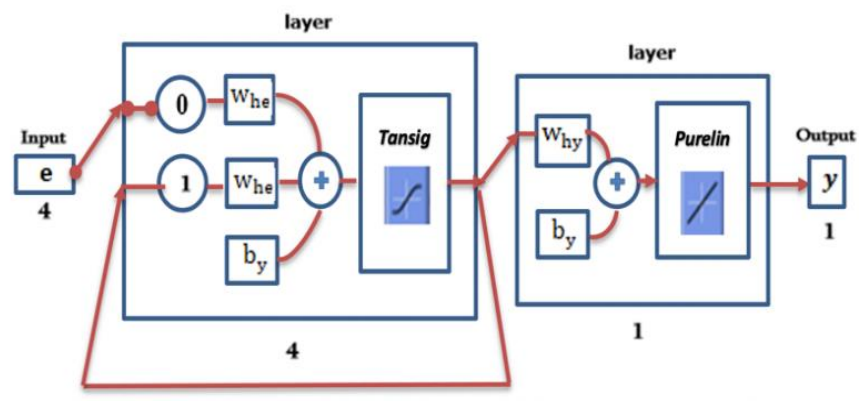

Figure 3. Layered Recurrent Neural Network construction

It can be shown from Figure 3 that the L-RNN consists of an input layer, a hidden layer, an output layer, and one supplementary background layer where the feedback output from the hidden layer have returned to hidden layer. The tangent sigmoid function (Eq. (1)) is activated in the hidden layer and the linear transfer activation function (Eq. (2)) is used at output layer.

Equation 1. Hyperbolic Tangent-Sigmoid Function

$$
\text { tansig }=\frac{2}{1+e^{-2 n}}-1
$$

Equation 2. Linear Transfer Function

$$
\operatorname{purelin}(n)=\left\{\begin{array}{cc}
n, & \text { if } n \geq 0 \\
-n, & \text { if } n<0
\end{array}\right.
$$

Set an input e vector as $\mathrm{e}=\left[\mathrm{e}_{1}, \ldots \ldots \ldots, \mathrm{e}_{\mathrm{t}}\right]$ which represents the extracted features. The constructed L-RNN calculates output vector $\mathrm{y}=\left[\mathrm{y}_{1}, \ldots \ldots \ldots, \mathrm{y}_{\mathrm{t}}\right]$ by updating Eq. (3).

\section{Equation 3. Neural Network Output}

$$
y_{t}=f\left(W_{h y} h_{t}+b_{y}\right)
$$

where, $f$ is sigmoid function, $\mathrm{W}_{\text {hy }}$ symbolizes the matrix of weights between hidden and output layer, $b_{y}$ is the bias factors vector and the hidden vector $h=\left[h_{1}, \ldots \ldots \ldots, h_{t}\right]$ can be using Eq. (4).

\section{Equation 4. Hidden Layers Output}

$$
h_{t}=f\left(W_{h e} e_{t}+W_{h h} h_{t-1}+b_{h}\right)
$$

where, the matrix $\mathrm{W}_{\text {he }}$ representsthe weights between the input and hidden layer, the matrix $\mathrm{W}_{\mathrm{hh}}$ represents the weights among the hidden layer with itself at nearby time levels and vector $b_{h}$ is bias factors. Many specific forms of training algorithms for L-RNN can be used. In this paper, quasi-newton backpropagation algorithm is implemented to train L-RNN by using 4 neurons in the hidden layer and 1000 epochs. In addition, the performance of L-RNN is evaluated using mean square error (MSE). Table 1 shows the parameters settings for L-RNN.

Table 1. L-RNN performance evaluation

\begin{tabular}{cc}
\hline Parameters & Values \\
\hline Maximum amount of iterations & 1000 \\
Neurons quantity in input layer & 4 \\
Neurons quantity in hidden layer & 4 \\
Neurons quantity in output layer & 1 \\
Maximum validation failures & 6 \\
Minimum performance gradient & $10^{-7}$ \\
Initial mutation & 0.001 \\
\hline
\end{tabular}

\section{RESULT AND ANALYSIS}

\subsection{Feature extraction results}

The proposed hybrid technique is implemented on MUCT database [36]. The MUCT database has 3755 human face with 76 manual landmarks. The result of extracted features using proposed method is shown in Table 2 .

Table 2 shows that each facial image is represented by a unique and small size feature column. This small size features column makes the extraction process time efficiently short. According to Table 2, the average time of the feature extraction process for one image is 0.06 seconds. Thus, for 1000 faces, the feature extraction process will require one minute, which is remarkably a short processing time. All images' facial features are then arranged in a matrix such that each image is represented by a column of four features as shown in Table 3. 
Table 2. Obtained feature elements and assign their classes

\begin{tabular}{|c|c|c|c|c|}
\hline $\begin{array}{c}\text { Class } \\
\text { No }\end{array}$ & $\begin{array}{l}\text { Image } \\
\text { index }\end{array}$ & Image & Features & $\begin{array}{l}\text { Time } \\
(\mathrm{sec})\end{array}$ \\
\hline 1 & i000qa-fn & & $\begin{array}{c}20932 \\
6168 \\
5534 \\
24962\end{array}$ & 0.0766 \\
\hline 2 & i001ra-mn & & $\begin{array}{c}16734 \\
5894 \\
6099 \\
28869\end{array}$ & 0.0409 \\
\hline 3 & i002ra-mn & & $\begin{array}{c}18482 \\
5851 \\
5883 \\
27380\end{array}$ & 0.0460 \\
\hline 4 & i004ra-mn & & $\begin{array}{c}16724 \\
5556 \\
5984 \\
29332\end{array}$ & 0.0419 \\
\hline 5 & i004ra-mn & & $\begin{array}{c}16929 \\
5509 \\
5818 \\
29340\end{array}$ & 0.0477 \\
\hline 6 & i005ra-fn & & $\begin{array}{c}18632 \\
6242 \\
5664 \\
27058\end{array}$ & 0.0446 \\
\hline 7 & i006ra-mn & & $\begin{array}{c}18002 \\
5538 \\
6119 \\
27937\end{array}$ & 0.0638 \\
\hline 8 & i007ra-fn & & $\begin{array}{c}17958 \\
5680 \\
6146 \\
27812\end{array}$ & 0.0558 \\
\hline 9 & i008ra-mn & & $\begin{array}{c}16976 \\
5431 \\
6020 \\
29169\end{array}$ & 0.0610 \\
\hline 10 & i009ra-mn & & $\begin{array}{c}17779 \\
5218 \\
5905 \\
28694\end{array}$ & 0.1210 \\
\hline 11 & i010qa-mn & & $\begin{array}{c}18834 \\
6070 \\
6184 \\
26508\end{array}$ & 0.1458 \\
\hline 12 & i011qa-mn & & $\begin{array}{c}17643 \\
6406 \\
6417 \\
27130\end{array}$ & 0.1458 \\
\hline
\end{tabular}

Table 3. Columns of four features for images representation

\begin{tabular}{ccc}
\hline Column 1 & Column 2 & Column 3 \\
\hline 20932 & 16734 & 18482 \\
6168 & 5894 & 5851 \\
5534 & 6099 & 5883
\end{tabular}

\begin{tabular}{ccc}
24962 & 28869 & 27380 \\
\hline Column 4 & Column 5 & Column 6 \\
\hline 16724 & 16929 & 18632 \\
5556 & 5509 & 6242 \\
5984 & 5818 & 5664 \\
29332 & 29340 & 27058 \\
\hline Column 7 & Column 8 & Column 9 \\
\hline 18002 & 17958 & 16976 \\
5538 & 5680 & 5431 \\
6119 & 6146 & 6020 \\
27937 & 27812 & 29169 \\
\hline Column 10 & Column 11 & Column 12 \\
\hline 17779 & 18834 & 17643 \\
5218 & 6070 & 6406 \\
5905 & 6184 & 6417 \\
28694 & 26508 & 27130 \\
\hline
\end{tabular}

\subsection{L-RNN results}

The classification process is performed on the database of extracted features using different neural networks that have the tangent sigmoid function in the hidden layer and the linear transfer activation function at output layer. Table 4 shows the result of implementing different types of ANN on extracted features for classification purpose. The training time, the number of iteration and the MSE are central aspects influencing the appropriateness of a classification algorithm. It can be noticed from Table 4 that L-RNN has the fastest training time $(1.7818 \mathrm{sec})$ and the lowest number of iterations (139). Though, MSE of L-RNN comes in the second position after the Cascade-Forward Backpropagation Network, the LRNN converges 21 times faster, which makes the L-RNN more reliable, feasible, and efficient in terms of training time and computation power. In addition, different training methods were tested and studied on the proposed technique. Table 5 illustrates results of using different training methods to train L-RNN. The results show that the quasi-Newton backpropagation gave the best result comparing with other training algorithms in terms of performance, training time, and number of iteration. At every iteration of the algorithm, quasi-Newton algorithm adjust an approximate Hessian matrix. The adjustment is calculated according to gradient. Figure 4 shows the performance of the L-RNN using quasi-Newton backpropagation in terms of MSE. From Table 5 and Figure 4, the best training performance $\left(6.38 * 10^{-23}\right)$ was achieved after just 64 epochs. These number of epochs are gradient descent hyper-parameter which responsible of managing the amount of total loops that update the parameters of the network. Hence, by using quasi-Newton back-propagation the number of epochs is reduced from 139 to 64. Therefore, the rate of convergence is improved. Figure 5 shows the gradient, mutation and validation checks of L-RNN for 64 epochs.

\subsection{Classification results}

The classification process based on the extracted features and the output of L-RNN was labeled with class hosting within database indices. The labeled classifier was trained and tested using L-RNN. The classification test reached up to $98 \%$ accuracy with no false acceptance rate found in the term of receiver operating characteristic. Figure 6 shows that the proposed algorithm identifies face image 7 as a class 7 .

The proposed technique achieved higher classification rate than other conventional methods as shown in Table 6 . 
Table 4. Implementation results of different ANN

\begin{tabular}{cccccc}
\hline Parameters & $\begin{array}{c}\text { Feed-Forward back } \\
\text { propagation network }\end{array}$ & $\begin{array}{c}\text { Function fitting } \\
\text { neural network }\end{array}$ & $\begin{array}{c}\text { Cascade-Forward back } \\
\text { propagation network }\end{array}$ & $\begin{array}{c}\text { EIman back } \\
\text { propagation network }\end{array}$ & $\begin{array}{c}\text { Layer recurrent } \\
\text { neural network }\end{array}$ \\
\hline $\begin{array}{c}\text { Training Time } \\
\text { Number of } \\
\text { iterations }\end{array}$ & 2.3938 & 4.3182 & 13.809 & 2.2079 & 1.7818 \\
$\begin{array}{c}\text { Performance } \\
\text { (MSE) }\end{array}$ & 291 & 815 & 2935 & 210 & 139 \\
Gradient & $2.1 * 10^{-20}$ & 7.82 & $1.56^{*} 10^{-24}$ & $7.64 * 10^{-17}$ & $2.63 * 10^{-22}$ \\
Mu & $6.04 * 10^{-9}$ & $9.88^{*} * 10^{-8}$ & $2.89^{-8} * 10^{-12}$ & & \\
$1 * 10^{-11}$ & $1 * 10^{-10}$ & $1 * 10^{-12}$ & $1 * 10^{-8}$ & $1.56810^{-11}$ \\
1 & & & $1 * 10^{-9}$ & \\
\hline
\end{tabular}

Table 5. Implementation results of L-RNN using various types of training algorithms

\begin{tabular}{cccccc}
\hline Parameters & $\begin{array}{c}\text { Gradient descent } \\
\text { with momentum }\end{array}$ & $\begin{array}{c}\text { Levenberg- } \\
\text { Marquardt back } \\
\text { propagation }\end{array}$ & $\begin{array}{c}\text { Quasi-Newton } \\
\text { back propagation }\end{array}$ & $\begin{array}{c}\text { Conjugate gradient } \\
\text { back propagation with } \\
\text { Polak-Ribiere updates }\end{array}$ & $\begin{array}{c}\text { RPROP back } \\
\text { propagation }\end{array}$ \\
\hline Performance & 5.24 & $5.06^{*} 10^{-22}$ & $6.38^{*} 10^{-23}$ & 3.83 & 0.879 \\
Time & 30.214 & 7.8396 & 1.79 & 1.8961 & 29.922 \\
No of iterations & 10000 & 240 & 64 & 165 & 10000 \\
\hline
\end{tabular}

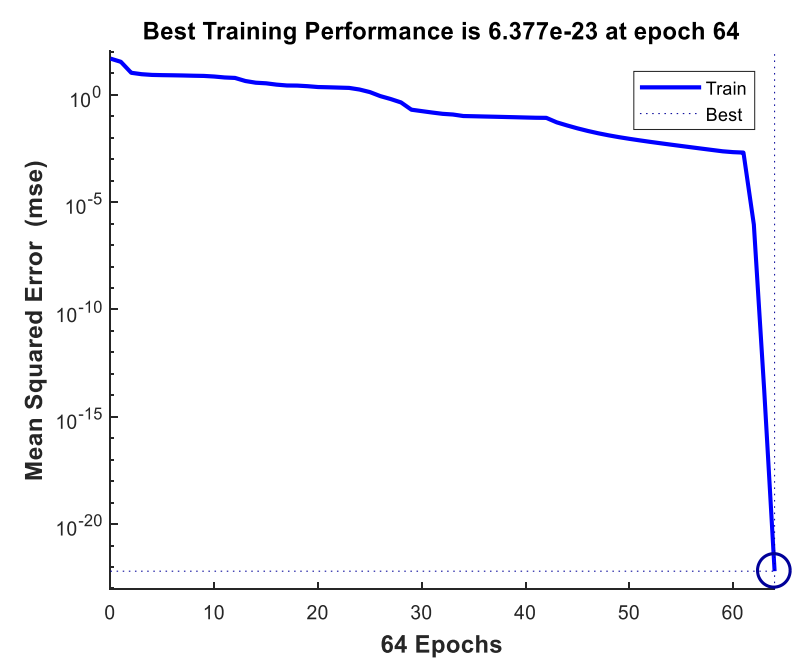

Figure 4. MSE of L-RNN using quasi-Newton backpropagation

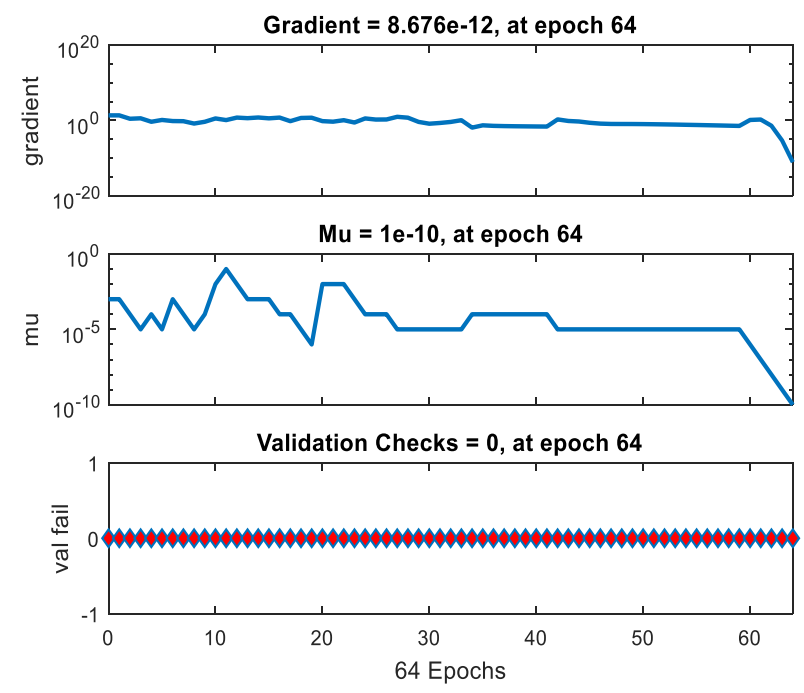

Figure 5. Gradient, mutation and validation checks of LRNN at 64 iteration

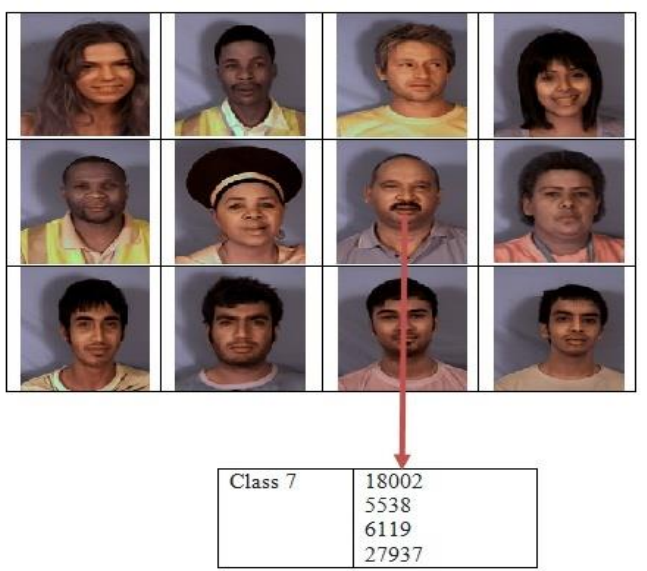

Figure 6. Image classification within proposed algorithm

Table 6. Classification rate comparision

\begin{tabular}{cc}
\hline Method & Rate \\
\hline Principle Component Analysis & $90 \%$ \\
Bilinear CNNs & $80-90 \%$ \\
Retinal Connected Neural Network & $79-90 \%$ \\
Rotations Invariant Neural Network (RINN) & $85-95 \%$ \\
Multi-Layer Perceptron (MLP) neural network & $96 \%$ \\
Proposed Hybrid Method & $98 \%$ \\
\hline
\end{tabular}

\section{CONCLUSIONS}

A Hybrid technique based on modified local binary pattern (MLBP) and Layered-Recurrent network (L-RNN) for human face recognition was proposed, implemented and tested on MUCT database. The reduction size of extracted features was achieved using MLBP. Hence, the needed time for feature extraction process was significantly reduced. The classification process was accomplished using a small size LRNN with quasi-Newton back propagation training algorithm. The Hybrid technique was performed superiorly over conventional techniques in terms of accuracy, recognition rate and number of iterations. Hence, the best training performance $\left(6.38 * 10^{-23}\right)$ was achieved after just 64 epochs with recognition rate $98 \%$. 


\section{REFERENCES}

[1] Wu, Y.M., Wang, H.W., Lu, Y.L., Yen, S., Hsiao, Y.T. (2012). Facial feature extraction and applications: A review. Berlin, Heidelberg, 2012: Springer Berlin Heidelberg, in Intelligent Information and Database Systems, 228-238. https://doi.org/10.1007/978-3-64228487-8 23

[2] Wang, H., Hu, J., Deng, W. (2018). Face feature extraction: A complete review. IEEE Access, 6: 60016039. https://doi.org/10.1109/ACCESS.2017.2784842

[3] Chitaliya, N.G., Trivedi, A.I. (2010). An efficient method for face feature extraction and recognition based on contourlet transforms and principal component analysis. Procedia Computer Science, 2: 52-61. https://doi.org/10.1016/j.procs.2010.11.008.

[4] Bay, H., Ess, A., Tuytelaars, T., Gool, L.V. (2008). Speeded-Up Robust Features (SURF). Comput. Vis. Image Underst., 110: 346-359. https://doi.org/10.1016/j.cviu.2007.09.014

[5] Purandare, V., Talele, K.T. (2014). Efficient heterogeneous face recognition using Scale Invariant Feature Transform. 2014 International Conference on Circuits, Systems, Communication and Information Technology Applications (CSCITA), Mumbai, 2014 https://doi.org/10.1109/CSCITA.2014.6839277

[6] Lu, X., Duan, X., Mao, X., Li, Y., Zhang, X. (2017). Feature extraction and fusion using deep convolutional neural networks for face detection. Mathematical Problems in Engineering, 2017: 1-9. https://doi.org/10.1155/2017/1376726.

[7] Liu, L., Fieguth, P., Guo, Y., Wang, X., Pietikäinen, M. (2017). Local binary features for texture classification: Taxonomy and experimental study. Pattern Recognition, 62: $135-160$ https://doi.org/10.1016/j.patcog.2016.08.032

[8] Azriansyah, E.M., Hartuti, N., Fachrurrozi, M., Tama B.A. (2019). A study about principle component analysis and eigenface for facial extraction. Journal of Physics: Conference Series, 1196. https://doi.org/10.1088/17426596/1196/1/012010

[9] Huang, D., Shan, C., Ardabilian, M., Wang, Y., Chen, L. (2011). Local binary patterns and its application to facial image analysis: A survey. IEEE Transactions on Systems, Man, and Cybernetics, Part C (Applications and Reviews), 41(6): 765-781. https://doi.org/10.1109/TSMCC.2011.2118750

[10] Meena, K., Suruliandi, A. (2011). Local binary patterns and its variants for face recognition. International Conference on Recent Trends in Information Technology (ICRTIT), Chennai, Tamil Nadu, pp. 782-786. https://doi.org/10.1109/ICRTIT.2011.5972286

[11] Lajevardi, S.M., Hussain, Z.M. (2012). Automatic facial expression recognition: Feature extraction and selection. Signal, Image and Video Processing, 6: 159-169. https://doi.org/10.1007/s11760-010-0177-5

[12] AlQaisi, A., AlTarawneh, M., Alqadi, Z.A., Sharadqah, A.A. (2019). Analysis of color image features extraction using texture methods. Telkomnika, 17(3): 1220-1225. http://dx.doi.org/10.12928/telkomnika.v17i3.9922

[13] Al-Qaisi, A., Khawatreh, S.A., Sharadqah, A.A., Alqadi, Z.A. (2018). Wave file features extraction using reduced LBP. International Journal of Electrical and Computer Engineering (IJECE), 8(5): 2780-2787. http://doi.org/10.11591/ijece.v8i5.pp2780-2787

[14] Al-Qaisi, A., Manasreh, A., Sharadqeh, A., Alqadi, Z. (2019). Digital color image classification based on modified local binary pattern using neural network. International Journal on Communications Antenna and Propagation, 9(6): 403-408. https://doi.org/10.15866/irecap.v9i6.18425

[15] Al-Qaisi, A. (2020). Arabic word dependent speaker identification system using artificial neural network. International Journal of Circuits, Systems and Signal Processing, 14 : 290-295. https://doi.org/10.46300/9106.2020.14.41

[16] Le, T.H. (2011). Applying artificial neural networks for face recognition. Advances in Artificial Neural Systems, 2011: 673016. https://doi.org/10.1155/2011/673016

[17] Kasar, M.M., Bhattacharyya, D., Kim, T.H. (2016). Face recognition using neural network: A review. International Journal of Security and Its Applications, 10(3): 81-100. http://dx.doi.org/10.14257/ijsia.2016.10.3.08.

[18] Jindal, N., Kumar, V. (2013). Enhanced face recognition algorithm using PCA with artificial neural networks. International Journal of Advanced Research in Computer Science and Software Engineering, 3(6): 864-872.

[19] Li, H.X., Lin, Z., Shen, X., Brandt, J., Hua, G. (2015). A convolutional neural network cascade for face detection. 2015 IEEE Conference on Computer Vision and Pattern Recognition (CVPR), pp. 5325-5334. https://doi.org/10.1109/CVPR.2015.7299170

[20] Yoo, S.H., Oh, S.K., Pedrycz, W. (2015). Optimized face recognition algorithm using radial basis function neural networks and its practical applications. Neural Netw, 69: 111-125. https://doi.org/10.1016/j.neunet.2015.05.001

[21] Lin, T.Y., RoyChowdhury, A., Maji, S. (2015). Bilinear CNN models for fine-grained visual recognition. Proceedings of the 2015 IEEE International Conference on Computer Vision (ICCV), 2015: IEEE Computer Society, pp. 1449-1457. https://doi.org/10.1109/ICCV.2015.170.

[22] Sun, T.H., Tien, F.C. (2008). Using backpropagation neural network for face recognition with $2 \mathrm{D}+3 \mathrm{D}$ hybrid information. Expert Systems with Applications, 35(1): 361-372. https://doi.org/10.1016/j.eswa.2007.07.059

[23] Markopoulos, A.P., Georgiopoulos, S., Manolakos, D.E. (2016). On the use of back propagation and radial basis function neural networks in surface roughness prediction. Journal of Industrial Engineering International, 12(3): 389-400. https://doi.org/10.1007/s40092-016-0146-x

[24] Zhang, Y., Shang, K., Wang, J., Li, N., Zhang, M.M.Y. (2018). Patch strategy for deep face recognition. IET Image Processing, 12(5): 819-825. http://dx.doi.org/10.1049/iet-ipr.2017.1085

[25] AL-Allaf, O.N.A. (2014). Review of face detection systems based artificial neural networks algorithms. The International Journal of Multimedia \& Its Applications (IJMA), 6(1): 1-16. http://dx.doi.org/10.5121/ijma.2014.6101

[26] Wiegand, S., Igel, C., Handmann, U. (2004). Evolutionary multi-objective optimisation of neural networks for face detection. International Journal of Computational Intelligence and Applications, 4(3): 237253. https://doi.org/10.1142/S1469026804001288

[27] Boughrara, H., Chtourou, M., Ben Amar, C., Chen, L. (2016). Facial expression recognition based on a MLP 
neural network using constructive training algorithm. Multimedia Tools and Applications, 75(2): 709-731. https://doi.org/10.1007/s11042-014-2322-6

[28] Divya, A., Raja, K.B., Venugopal, K.R. (2019). Sorting pixels based face recognition using discrete wavelet transform and statistical features. 2019 3rd International Conference on Imaging, Signal Processing and Communication (ICISPC), pp. 150-154. https://doi.org/10.1109/ICISPC.2019.8935675

[29] Pan, Z.B., Li, Z., Fan, H., Wu, X. (2017). Feature based local binary pattern for rotation invariant texture classification. Expert Systems with Applications, 88: 238-248. https://doi.org/10.1016/j.eswa.2017.07.007

[30] Nannia, L., Luminib, A., Brahnam, S. (2012). Survey on LBP based texture descriptors for image classification. Expert Systems with Applications, 39(3): 3634-3641. https://doi.org/10.1016/j.eswa.2011.09.054

[31] Ojala, T., Pietikainen, M., Maenpaa, T. (2002). Multiresolution gray-scale and rotation invariant texture classification with local binary patterns. IEEE Transactions on Pattern Analysis and Machine Intelligence, 24(7): 971-987.
https://doi.org/10.1109/TPAMI.2002.1017623

[32] Singh, C., Walia, E., Kaur, K.P. (2018). Color texture description with novel local binary patterns for effective image retrieval. Pattern Recognition, 76: 50-68. https://doi.org/10.1016/j.patcog.2017.10.021

[33] Zhang, X.Y., Yin, F., Zhang, Y., Liu, C., Bengio, Y. (2018). Drawing and recognizing Chinese characters with recurrent neural network. IEEE Transactions on Pattern Analysis and Machine Intelligence, 40(4): 849862. https://doi.org/10.1109/TPAMI.2017.2695539

[34] Ruiz, L.G.B., Rueda, R., Cuéllar, M.P., Pegalajar, M.C. (2018). Energy consumption forecasting based on Elman neural networks with evolutive optimization. Expert Systems with Applications, 92: 380-389. https://doi.org/10.1016/j.eswa.2017.09.059

[35] Elman, J.L. (1990). Finding structure in time. Cognitive Science, 14(2): 179-211. https://doi.org/10.1016/03640213(90)90002-E

[36] Milborrow, S., Morkel, J., Nicolls, F. The MUCT Landmarked Face Database. PRASA. http://www.milbo.org/muct/, accessed on Mar. 17, 2020. 